\title{
Effect of increasing the delivery of smoking cessation care in alcohol and other drug treatment centres: a cluster-randomized controlled trial
}

\author{
Ashleigh Guillaumier, ${ }^{1,9}$, Eliza Skelton ${ }^{1,9}$ (D), Anthony Shakeshaft ${ }^{2}$, Michael Farrell ${ }^{2}$ (D), \\ Flora Tzelepis ${ }^{1,3}$ (D), Scott Walsberger ${ }^{4}$, Catherine D'Este ${ }^{5,1}$, Christine Paul ${ }^{1,9}$, Adrian Dunlop ${ }^{1,6}$, \\ Robert Stirling ${ }^{7}$, Carrie Fowlie ${ }^{8}$, Peter Kelly ${ }^{10}$, Christopher Oldmeadow ${ }^{9}$, Kerrin Palazzi ${ }^{9}$ \& \\ Billie Bonevski ${ }^{1,9}$ (iD
}

\section{ABSTRACT}

Aim Aims were to test the effectiveness of an organizational change intervention integrating smoking cessation treatment into usual alcohol and other drug (AOD) treatment, compared with usual care, on (1) 7-day point prevalence abstinence (PPA) at 8 weeks follow-up; (2) prolonged abstinence; (3) cigarettes smoked per day; (4) number of quit attempts; and (5) offer and use of nicotine replacement therapy (NRT). All outcomes were assessed at 8 weeks and 6.5 months follow-up. Design Cluster-randomized controlled trial, with AOD service as unit of randomization, conducted January 2015-March 2016. Setting Thirty-two eligible services (provided face-to-face client sessions to $\geq 50$ clients/year) in Australia were randomized to control (usual care; $n=15$ ) or intervention $(n=17)$ groups by an independent blinded biostatistician. Participants Eligible participants ( $\geq 16$ years, current smoker) completed surveys at the service at baseline ( $n=896)$ and telephone follow-up surveys (conducted by blinded assessors) at 8 weeks ( $n=471 ; 53 \%)$ and 6.5 months $(n=427 ; 48 \%)$. Intervention Intervention services received an intervention to establish routine screening, assessment and delivery of smoking cessation care. Measurements Primary outcome was biochemically verified 7-day PPA at 8week follow-up. Secondary outcomes included verified and self-reported prolonged abstinence, self-reported 7-day PPA, cigarettes/day, quit attempts and offer and use of NRT. Intention-to-treat analyses were performed, assuming missing participants were not abstinent. Findings At 8 weeks, the findings in verified 7-day PPA between groups [2.6 versus 1.8\%, odds ratio $(\mathrm{OR})=1.72,95 \%$ confidence interval $(\mathrm{CI})=0.5-5.7, P=0.373]$ were inconclusive as to whether a difference was present. Significantly lower mean cigarettes/day were reported in the intervention group compared to the usual care group at 8 weeks [incidence rate ratio $(\mathrm{IRR})=0.88,95 \% \mathrm{CI}=0.8-0.95, P=0.001$ ] but were similar at 6.5 months $(\mathrm{IRR}=0.96,95 \% \mathrm{CI}=0.9-1.02, P=0.240)$ follow-up. At both follow-ups the intervention group reported higher rates of NRT use. Conclusions Integrating smoking cessation treatment into addiction services did not significantly improve short-term abstinence from smoking.

Keywords Cessation, cluster randomized controlled trial, intervention, organizational change, smoking, tobacco.

Correspondence to: Billie Bonevski, Faculty of Health and Medicine, School of Medicine and Public Health, The University of Newcastle, 1 University Drive, Level 5 McAuley Building, Calvary Mater Hospital, Waratah, NSW 2298, Australia. E-mail: billie.bonevski@newcastle.edu.au

Submitted 13 May 2019; initial review completed 25 July 2019; final version accepted 15 November 2019

\section{INTRODUCTION}

The prevalence of smoking among people in alcohol and other drug (AOD) treatment is two to five times higher [1] than that in the general population $[2,3]$. The consequences of the high smoking rates are devastating, with tobacco-related diseases such as cancer, cardiovascular disease and chronic respiratory disease the leading causes of death in this population [4].
Despite interest in quitting and frequent quit attempts [5-7], successful long-term quit rates among people in AOD treatment are much lower than those in the general population of smokers [8]. High relapse rates in this population may be due to factors related to addiction (e.g. smoking related cues and triggers) $[9,10]$, lack of cessation support [11-13], nicotine dependence and the high levels of smoking in their social network [14]. Smoking cessation trials indicate that if given sufficient support to quit, people 
in AOD treatment can achieve cessation, even if only shortterm, and that smoking cessation enhances rather than hinders the achievement of other drug treatment goals [15].

Provision of evidence-based smoking cessation treatment in AOD services is suboptimal [16]. Although clinically recommended for all people in AOD treatment [17], a minority have their smoking status assessed, are given brief advice to quit, offered nicotine replacement therapy (NRT) or other pharmacotherapies and referred to other cessation support, such as telephone quitlines [16,18].

Barriers to the provision of this care include a lack of staff training and confidence, a smoking-permissive history and culture, lack of systems to address smoking cessation, high staff smoking rates and misperceptions among staff, such as addressing smoking may jeopardize other treatment effectiveness $[16,19,20]$. To address these barriers, multi-component, whole-of-organization approaches are needed [21]. The organizational change approach tested in the current study draws from two frameworks: the Addressing Tobacco Through Organizational Change (ATTOC) model $[22,23]$ and the Systems Change approach [21] for the integration of smoking cessation treatment into health services. Both frameworks outline at least six core components, including organizational leadership, site-based champions or working group, electronic or paper-based systems for identifying smokers and recording provision of support to quit, training for staff, free-of-charge resources and evidence-based treatment provision to people who smoke [21-23]. To date, no randomized controlled trials have been conducted to test the effectiveness of organizational change approaches for achieving abstinence among people in AOD treatment services [24].

Small pilot studies in the United States [22,23] suggest that organizational change following the ATTOC and Systems Change approaches is acceptable to staff and clients, and shows promise at increasing the delivery of smoking cessation care within AOD treatment services [25]. Two systematic reviews of organizational change interventions for smoking cessation have been published, showing similar outcomes. A Cochrane Review by Thomas et al. [26] of seven randomized controlled trials describing organizational change interventions in a variety of health-care settings found evidence for improvements in the provision of smoking cessation care (improvements in documentation of smoking status, quitline referral and quitline enrolment, but no evidence of impact on smoking cessation rates. Also, no studies implemented all six components of organizational change. No studies set in the AOD sector were included. The second review, by Skelton et al. [24], included 14 papers describing seven organizational change intervention studies in the AOD setting and found similar results, improvements in smoking cessation practices and little evidence of reductions of smoking rates in people attending treatment. However, no cluster randomized controlled trials were found. Thus, research to date suggests that while organizational change interventions may be effective at changing clinical practice, increasing delivery of smoking cessation care, less is known about the impact on the smoking rates of people in treatment. Both reviews noted the lack of implementation of all components of organizational change interventions, with most studies describing two to four of the possible six components. We designed the current trial to address the lack of randomized controlled trials of organizational change for smoking cessation reporting use of all six intervention components and examining client smoking cessation outcomes in AOD treatment services. Pilot research informed the design of the current trial. A single-site, pre- to post-intervention, mixed-methods study showed that staff-reported smoking cessation practices significantly increased for the provision of verbal advice (30-82\%), offer of free or subsidized nicotine replacement therapy (30-91\%), referral to a general practitioner (19-64\%) and follow-up to check on quit smoking progress (18.5-64\%). More than $85 \%$ of staff agreed that it was acceptable to address smoking as part of usual care and $95 \%$ of people in treatment agreed that it was acceptable to be asked by staff about their tobacco smoking [25,27]. However, no randomized controlled trials have been conducted of the effectiveness of the approach at improving cessation rates among smokers in AOD treatment. Given the whole-of-organization approach of the intervention, the randomization of individual participants is inappropriate and the cluster randomized controlled trial design is recommended.

The primary aim of the Tackling Nicotine Together (TNT) trial is to compare the effectiveness of an organizational change intervention in increasing smoking cessation [7-day point prevalence abstinence (PPA) biochemically verified at 8 weeks] with usual care among individuals attending AOD treatment services. Secondary aims of the trial are to compare with usual care, the intervention's effectiveness in increasing self-reported prolonged smoking abstinence, quit attempts and receipt and use of NRT among individuals attending AOD treatment services at 8 weeks and 6.5 months follow-up. Although outcomes are collected from individual participants, the study aims to examine changes at cluster/service level.

\section{METHODS}

\section{Study design}

A cluster randomized controlled trial with 32 governmental and non-government AOD treatment services in four mainland states or territories of Australia [New South Wales (NSW), Australian Capital Territory (ACT), Queensland (QLD) and South Australia (SA)] was conducted between January 2015 and March 2016. The AOD services 
(clusters) were the unit of randomization. The full study protocol has been described in detail [28]. Briefly, following randomization, all 32 AOD services assisted with the recruitment of their clients into the baseline survey over a 6-month period. An independent computer-assisted telephone interviewing (CATI) team, blinded to participant's group allocation, conducted follow-up telephone client surveys 8 weeks and 6.5 months later, following a 2 -week grace period post-baseline survey.

\section{Recruitment of treatment centres (clusters)}

Eligible AOD services were those providing treatment involving at least one face-to-face client session to at least 50 clients per year. Recruitment was conducted through key contacts (such as Directors of Health Services or peak non-governmental organizations). Treatment services were invited to participate via a call for expressions of interest and followed-up by telephone and in person by the TNT research manager to determine eligibility.

\section{Randomization of treatment centres}

Recruited trial centres that consented into the project were randomized into groups following recruitment following recruitment of all sites. Randomization was undertaken by an independent statistician who used a combination of stratified and restricted randomization to ensure equitable distribution across the intervention groups of stratification factors including state/territory (NSW, QLD, ACT and SA), service type (governmental or non-government organization) and type of smoking policy (partial or total smoking ban). Randomization was undertaken using generation of random uniform values. The randomization process is described in detail elsewhere [28].

\section{Concealment of allocation}

The trial was partially blinded. Treatment services were the unit of randomization, and staff were aware of allocation once intervention implementation commenced. Participating clients were not aware of allocation, as they were simply asked to complete a survey about smoking on three occasions. The follow-up assessments were conducted by independent telephone interviewers who were blind to allocation when assessing the primary and secondary outcomes.

\section{Participants and data collection}

People in treatment eligible for inclusion in the trial were aged 16 years and over and attending their first of a number of visits (to allow for repeated exposure to the intervention), were self-reported current smokers, sufficiently proficient in English and were cognitively and mentally able to give informed consent according to service staff who approached participants. Participants provided written informed consent after receiving a complete description of the study. Consenting participants completed a baseline survey via a touchscreen tablet at the time of recruitment and an additional two telephone surveys 8 weeks and 6.5 months later (allowing for a 2-week grace period post-baseline). At the 8-week and 6.5-month follow-up assessments, participants who reported 7-day PPA were asked to return to the service to provide a breath sample to measure carbon monoxide (CO). A number of retention strategies were used to minimize loss to follow-up [28].

\section{Interventions}

The intervention was delivered to entire treatment services (clusters) in the intervention group. An e-mail to all services notifying randomization allocation marked the study-wide commencement of the intervention period. The continuous 12-week intervention period occurred during the 6 weeks prior to commencement of client recruitment and for a further 6 weeks during client recruitment. In the first half of the intervention period control services observed a 6-week 'waiting period' during which they had no study-related obligations. Meanwhile, intervention services were sent electronic copies of all intervention materials and patient education resources and were scheduled a 1-day skills training work-shop together with a visit from the study team to deliver the NRT and hardcopy intervention resources. Intervention services continued to receive support for organizational change activities for a further 6 weeks after client recruitment had commenced.

\section{Intervention group}

During a 12-week period, treatment services in the intervention group participated in an 'organizational change' intervention which aimed to establish or improve routine screening, assessment and delivery of best-practice smoking cessation care and treatment. The intervention included eight core components to support changing the culture and performance of the service to include more smoking cessation support for people who smoke: (1) engage organizational support [29]; (2) identify and support a champion [21,22]; (3) promote centre policies that support and provide tobacco dependence services [29,30]; (4) implement a system of identifying smokers [31]; (5) provide education and resources [21,32]; (6) provide staff and client feedback regarding smoke-free policy and smoking cessation care and support [33]; (7) use of evidence-based cessation treatments [34]; and (8) maintenance and follow-up [29]. A brief intervention description appears in Supporting information, Table S1; a full description appears in the published protocol paper [28]. 
Usual care group

Treatment services in the usual care group were not provided with the intervention and asked to maintain usual care.

\section{Outcomes}

The primary outcome was individual client smoking cessation at 8-week follow-up using biochemically verified 7-day PPA. The 7-day PPA was assessed at 8 weeks and 6.5 months using standard self-reported items verified with measures of $\mathrm{CO}$ in expired air to determine whether participants had smoked any tobacco [CO cut-off 8 parts per million (p.p.m.)] in the preceding 7 days $[35,36]$.

Secondary outcomes were: prolonged abstinence, assessed using a modified Russell Standard criteria [37] using the question: 'in the last 6 weeks/ 6 months have you smoked a cigarette, even a puff', determined at the 8-week follow-up and confirmed at the 6.5-month followup, taking into account an initial 2-week grace period. The grace period was used instead of the usual Russell Standard allowance for five cigarettes smoked. Selfreported 7-day-PPA; nicotine dependence (two-item HSI) [38]; number of cigarettes smoked per day; self-reported quit attempts in the last 6 weeks/ 6 months; and selfreported offer and use of NRT were collected at client 8week and 6.5-month follow-ups.

\section{Sample size}

Within each participating cluster, recruitment was expected to occur over a continuous 6-month period, with the aim of recruiting 35 participants per week (approaching 60 clients with $60 \%$ consent $[39,40]$ and giving a sample size at baseline of approximately 450 per group. Although strategies were employed to reduce attrition and maximize retention, based on previous research [41,42] $40 \%$ were expected to be lost to follow-up at 8 weeks, thereby providing a sample of 270 participants per experimental arm (an average of 16-17 per treatment centre). Assuming a 5\% significance level, $80 \%$ power, $5 \%$ smoking cessation in the control group for 7-day point prevalence, a design effect of 1.4 due to correlation of observations within treatment services (an intraclass correlation coefficient (ICC) of approximately 0.025) and a 10\% allowance for unequal-sized clusters, this sample allowed detection of a $9 \%$ difference in the 7 -day point prevalence of abstinence between groups at 8 weeks.

\section{STATISTICAL METHODS}

The statistical analysis plan was approved by all investigators before unblinding (see Supporting information, Appendix S1). Mixed-effects regression models were used to compare the primary and secondary outcomes between the two groups at each of the two follow-up times: logistic mixed modelling for binary outcomes, linear mixed modelling for HSI and negative binomial mixed modelling for the number of quit attempts and number of cigarettes smoked. A random intercept for site is included in crude and adjusted modelling to adjust for correlation of outcomes within treatment centres (clusters). Adjusted models include type of smoking policy (total versus partial/none; stratification variable), HSI and type of programme (residential-rehabilitation versus other) as fixed effects; adjusted modelling for count outcomes (cigarettes/day and quit attempts) also include baseline count as a fixed effect. Crude count (\%) or mean values [standard deviation (SD)] and crude and adjusted effect sizes [odds ratio (OR) or incidence rate ratio (IRR]) with 95\% confidence intervals (CIs) and $P$-values are presented.

An intention-to-treat (ITT) analysis was performed. Missing data at follow-up were imputed using single imputation methods: assuming those with no follow-up data are smokers [not PPA, per Society for Research on Nicotine and Tobacco (SRNT) guidelines and the Russell Standard], have CO p.p.m. above 8 (not verified PPA), have no change in number of cigarettes since baseline, have made no attempts to quit and are not taking NRT. Additional sensitivity analyses were performed:

1 Complete case analysis: modelling includes data for participants who have complete information.

2 Multiple imputation (MI) assuming missing not at random (MNAR): for the primary outcome of verified 7day PPA at 8 weeks only, assuming that missingness in the outcome is related to cessation status. Variables that are predictive of smoking status and missing data (age, gender, Indigenous status, education, housing, income, HSI, cannabis use, alcohol use, quit intentions, selfefficacy and motivation to quit) were used to create 10 imputed data sets; the proportion of participants with missing primary outcome who were non-smokers was set at (a) approximately $10 \%$ and (b) approximately $15 \%$ [43]. The analysis model was fitted to each of these imputed data sets and results pooled across imputed sets using Rubin's rules [44].

Statistical analyses were programmed using SAS version 9.4 (SAS Institute, Cary, NC, USA). A priori, $P<0.05$ (two-tailed) was used to indicate statistical significance.

\section{RESULTS}

Figure 1 shows the recruitment and randomization of service sites. Initially, 33 sites were recruited; one site withdrew following randomization but before commencement 


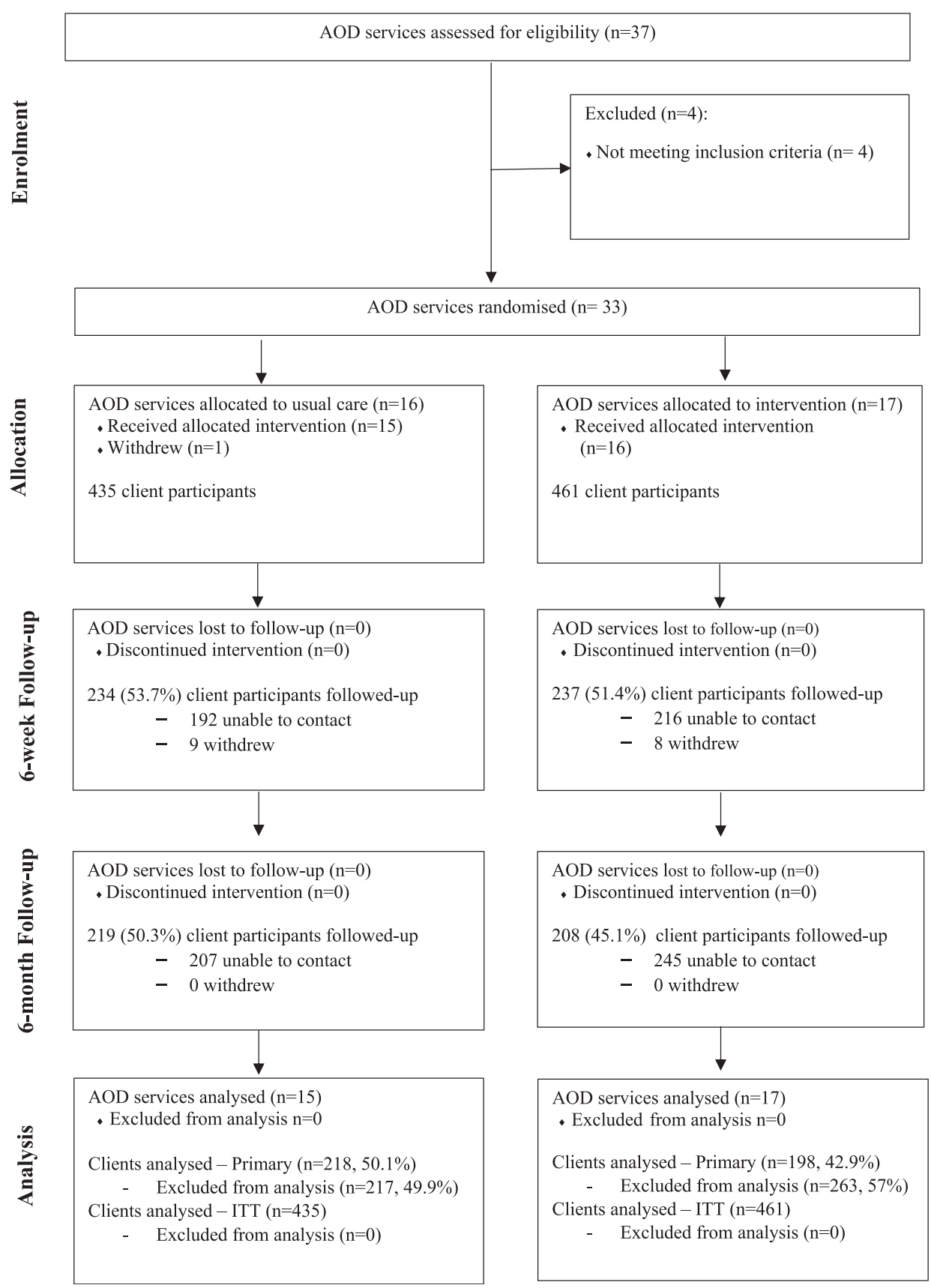

Figure I Consolidated Standards of Reporting Trials (CONSORT) flow diagram for alcohol and other drug (AOD) services AOD services allocated to intervention $(n=17)$

of participant recruitment due to changes in service management, resulting in 32 sites completing the trial. Service characteristics are well balanced between treatment groups (Table 1). A variety of AOD treatment programme types were included: residential rehabilitation/therapeutic community $(n=13,41 \%)$, out-patient counselling $(n=9,28 \%)$, opiate treatment/methadone maintenance $(n=7,22 \%)$, specialist detoxification unit $(n=2,6 \%)$ and a harm minimization service $(n=1,3 \%)$. Depending on programme type, average length of treatment for clients could be from 1 week to a number of months. Services addressed a number of substances, including heroin and other opioids, benzodiazepines, alcohol, amphetamines and cannabis. In total, 896 participants were recruited from the 32 sites nationally, 471 retained at 8 weeks (53\%) and 427 retained at 6.5 months (48\%). Attrition rates were not significantly different at 8 weeks $(P=0.475)$ and 6.5 months $(P=0.118)$.

Table 2 shows the baseline socio-demographic characteristics of participants and Table 3 shows the smoking 
Table 1 Service characteristics by treatment group, $n(\%)$.

\begin{tabular}{lcll}
\hline Characteristic & $\begin{array}{l}\text { Usual care } \\
(n=15)\end{array}$ & $\begin{array}{l}\text { Treatment } \\
(n=17)\end{array}$ & $\begin{array}{l}\text { Total } \\
(\mathrm{N}=32)\end{array}$ \\
\hline $\begin{array}{l}\text { State } \\
\text { New South Wales }\end{array}$ & $8(53 \%)$ & $9(53 \%)$ & $17(53 \%)$ \\
Queensland & $3(20 \%)$ & $4(24 \%)$ & $7(22 \%)$ \\
Australian Capital & $4(27 \%)$ & $4(24 \%)$ & $8(25 \%)$ \\
$\quad$ Territory & & & \\
Service & & & \\
$\quad$ Government & $6(40 \%)$ & $7(41 \%)$ & $13(41 \%)$ \\
$\quad$ Non-government & $9(60 \%)$ & $10(59 \%)$ & $19(59 \%)$ \\
$\quad$ organization & & & \\
Smoking policy & $7(47 \%)$ & $8(47 \%)$ & $15(47 \%)$ \\
$\quad \begin{array}{l}\text { Partial ban } \\
\text { Total ban }\end{array}$ & $8(53 \%)$ & $9(53 \%)$ & $17(53 \%)$ \\
Program type: residential rehabilitation & & \\
$\quad$ No & $9(60 \%)$ & $10(59 \%)$ & $19(59 \%)$ \\
$\quad$ Yes & $6(40 \%)$ & $7(41 \%)$ & $13(41 \%)$ \\
\hline
\end{tabular}

${ }^{a}$ Includes one service located in South Australia.

and other substance use characteristics. Treatment groups appear well balanced on all major characteristics, with the possible exceptions of quit intentions and quit confidence (Table 3).

Table 4 shows primary and secondary outcomes at 8-week and 6.5-month follow-up (see Supporting information, Table S2 for complete case results, and MI-MNAR analyses for the primary outcome). The findings were inconclusive as to whether or not a difference was present between the intervention and usual care groups in the primary outcome of verified 7-day PPA at 8-week followup. The ICC was found to be 0.27 ; approximately a quarter of the variability of the verified 7-day PPA can be explained by the between-site difference. Sensitivity analyses assuming higher rates of verified 7-day PPA in participants who did not return for follow-up also proved inconclusive regarding a difference between the intervention and usual care groups.

For the secondary cessation outcomes, the findings were inconclusive regarding treatment effect for selfreported 7-day PPA at either 8-week or 6.5-month follow-ups. The findings were also inconclusive as to whether a treatment effect was observed for either verified or self-reported prolonged abstinence outcomes at 8 weeks, and due to small cell sizes analyses could not be undertaken for the 6.5-month time-point.

Significantly lower mean cigarettes per day were observed in the intervention participants at 8 weeks, although this lost significance at 6.5 months. The findings were inconclusive regarding whether or not treatment effects were observed for number of quit attempts at either follow-up time-point.

The intervention group reported higher rates of NRT use overall and receipt of NRT at the clinic at both followup time-points.
Table 2 Participant demographics by treatment group, mean (SD) or $n(\%)$.

\begin{tabular}{|c|c|c|c|}
\hline Characteristic & $\begin{array}{l}\text { Usual care } \\
(n=435)\end{array}$ & $\begin{array}{l}\text { Treatment } \\
(n=461)\end{array}$ & $\begin{array}{l}\text { Total } \\
(N=896)\end{array}$ \\
\hline Age (years) & $37(11)$ & $37(11)$ & $37(11)$ \\
\hline \multicolumn{4}{|l|}{ Gender } \\
\hline Male & $264(61 \%)$ & $254(55 \%)$ & $518(58 \%)$ \\
\hline Female & $170(39 \%)$ & $203(44 \%)$ & $373(42 \%)$ \\
\hline \multicolumn{4}{|l|}{ Sexuality } \\
\hline Straight/heterosexual & $373(86 \%)$ & $393(85 \%)$ & $766(86 \%)$ \\
\hline $\begin{array}{r}\text { Lesbian, gay or } \\
\text { homosexual }\end{array}$ & $24(5.5 \%)$ & $19(4.1 \%)$ & $43(4.8 \%)$ \\
\hline Bisexual & $25(5.7 \%)$ & $24(5.2 \%)$ & $49(5.5 \%)$ \\
\hline Other & $6(1.4 \%)$ & $2(0.4 \%)$ & $8(0.9 \%)$ \\
\hline Prefer not to answer & $7(1.6 \%)$ & $22(4.8 \%)$ & $29(3.2 \%)$ \\
\hline \multicolumn{4}{|l|}{ Housing } \\
\hline Other & $252(58 \%)$ & $256(56 \%)$ & $508(57 \%)$ \\
\hline Own/rental house & $183(42 \%)$ & $205(44 \%)$ & $388(43 \%)$ \\
\hline \multicolumn{4}{|l|}{ Indigenous } \\
\hline Aboriginal and/or TSI & $54(12 \%)$ & $62(13 \%)$ & $116(13 \%)$ \\
\hline \multicolumn{4}{|l|}{ Education } \\
\hline Secondary or less & $305(70 \%)$ & $309(67 \%)$ & $614(69 \%)$ \\
\hline Tertiary qualifications & $129(30 \%)$ & $152(33 \%)$ & $281(31 \%)$ \\
\hline \multicolumn{4}{|l|}{ Income } \\
\hline$\leq \$ 400$ per week & $233(54 \%)$ & $262(57 \%)$ & $495(56 \%)$ \\
\hline$>\$ 400$ per week & $122(28 \%)$ & $122(27 \%)$ & $244(27 \%)$ \\
\hline Prefer not to answer & $78(18 \%)$ & $74(16 \%)$ & $152(17 \%)$ \\
\hline \multicolumn{4}{|l|}{ Main source of income } \\
\hline $\begin{array}{l}\text { Paid employment } \\
\text { (either full- or part- } \\
\text { time) }\end{array}$ & $80(18 \%)$ & $81(18 \%)$ & $161(18 \%)$ \\
\hline $\begin{array}{l}\text { Government pension } \\
\text { or benefit }\end{array}$ & $342(79 \%)$ & $361(79 \%)$ & $703(79 \%)$ \\
\hline Family member & $5(1.2 \%)$ & $8(1.7 \%)$ & $13(1.5 \%)$ \\
\hline Personal savings & $3(0.7 \%)$ & $5(1.1 \%)$ & $8(0.9 \%)$ \\
\hline Other & $4(0.9 \%)$ & $3(0.7 \%)$ & $7(0.8 \%)$ \\
\hline
\end{tabular}

TSI $=$ Torres Strait Islander; $\mathrm{SD}=$ standard deviation. ${ }^{\mathrm{a}}$ Response totals may not add to group or total $N$ due to missing data.

\section{DISCUSSION}

The results were inconclusive as to whether or not client verified abstinence rates at 8-week follow-up differed between conditions; however, a number of positive secondary outcomes were found. Reductions were seen in cigarettes smoked per day as well as increases in NRT offered at the service and used by participants at 8-week and 6.5-month follow-ups.

The study found very low verified smoking cessation rates among participants in both groups $(2.6 \%$ intervention and $1.8 \%$ control). This is not unusual in smoking cessation trials with AOD populations [45]. Although participants from intervention services were almost twice as likely as those in control services to achieve verified 7day abstinence at 8-week follow-up, the difference is not 
Table 3 Baseline smoking, drug use, and quit characteristics by treatment group, mean (SD) or $n(\%){ }^{\text {a }}$

\begin{tabular}{|c|c|c|c|}
\hline Characteristic & Usual care $(n=435)$ & Treatment $(n=461)$ & Total $(N=896)$ \\
\hline \multicolumn{4}{|l|}{ Smoking status } \\
\hline Yes, daily & $410(94 \%)$ & $446(97 \%)$ & $856(96 \%)$ \\
\hline Yes, at least once a week & $15(3.4 \%)$ & $6(1.3 \%)$ & $21(2.3 \%)$ \\
\hline Yes, but less often than once a week & $10(2.3 \%)$ & $9(2.0 \%)$ & $19(2.1 \%)$ \\
\hline Cigarettes per day & $19(11)$ & $19(11)$ & $19(11)$ \\
\hline \multicolumn{4}{|l|}{ Nicotine dependence $^{\mathrm{b}}$} \\
\hline Mean (SD) & $3(2)$ & $3(1)$ & $3(1)$ \\
\hline Low & $127(30 \%)$ & $115(26 \%)$ & $242(28 \%)$ \\
\hline Moderate & $209(49 \%)$ & $218(50 \%)$ & $427(50 \%)$ \\
\hline High & $89(21 \%)$ & $102(23 \%)$ & $191(22 \%)$ \\
\hline \multicolumn{4}{|l|}{ Product used } \\
\hline Cigarettes (commercial) & $330(79 \%)$ & $344(75 \%)$ & $674(77 \%)$ \\
\hline Roll your own & $291(69 \%)$ & $307(68 \%)$ & $598(68 \%)$ \\
\hline Loose tobacco & $25(6.3 \%)$ & $14(3.1 \%)$ & $39(4.6 \%)$ \\
\hline Cigars/pipe & $20(5.0 \%)$ & $18(4.0 \%)$ & $38(4.5 \%)$ \\
\hline Chewing tobacco & $3(0.8 \%)$ & $3(0.7 \%)$ & $6(0.7 \%)$ \\
\hline Snuff & $1(0.3 \%)$ & $3(0.7 \%)$ & $4(0.5 \%)$ \\
\hline Electronic cigarettes & $32(8.0 \%)$ & $30(6.7 \%)$ & $62(7.3 \%)$ \\
\hline Tobacco: other & $21(5.3 \%)$ & $16(3.6 \%)$ & $37(4.4 \%)$ \\
\hline Cannabis use & $195(45 \%)$ & $193(42 \%)$ & $388(43 \%)$ \\
\hline \multicolumn{4}{|l|}{ Tobacco and cannabis } \\
\hline No, never & $8(4.1 \%)$ & $7(3.6 \%)$ & $15(3.9 \%)$ \\
\hline Yes, sometimes & $23(12 \%)$ & $27(14 \%)$ & $50(13 \%)$ \\
\hline Yes, always & $164(84 \%)$ & $159(82 \%)$ & $323(83 \%)$ \\
\hline \multicolumn{4}{|l|}{ Drug use } \\
\hline Alcohol & $288(68 \%)$ & $305(67 \%)$ & $593(67 \%)$ \\
\hline Amphetamines & $162(38 \%)$ & $163(36 \%)$ & $325(37 \%)$ \\
\hline Hallucinogens & $20(4.8 \%)$ & $16(3.5 \%)$ & $36(4.1 \%)$ \\
\hline Inhalants & $21(5.0 \%)$ & $10(2.2 \%)$ & $31(3.6 \%)$ \\
\hline Opioids & $173(41 \%)$ & $196(43 \%)$ & $369(42 \%)$ \\
\hline Tranquillizers & $159(38 \%)$ & $158(34 \%)$ & $317(36 \%)$ \\
\hline Synthetic drugs & $41(9.8 \%)$ & $41(9.1 \%)$ & $82(9.4 \%)$ \\
\hline Drugs: other & $47(11 \%)$ & $50(11 \%)$ & $97(11 \%)$ \\
\hline Quit attempt ever & $340(78 \%)$ & $379(82 \%)$ & $719(80 \%)$ \\
\hline No. quit attempts (past 12 months) & $2(6)$ & $2(4)$ & $2(5)$ \\
\hline Motivation to quit & $6(3)$ & $7(3)$ & $6(3)$ \\
\hline \multicolumn{4}{|l|}{ Quitting intentions } \\
\hline Don’t know & $95(22 \%)$ & $56(12 \%)$ & $151(17 \%)$ \\
\hline Never quit & $13(3.0 \%)$ & $5(1.1 \%)$ & $18(2.0 \%)$ \\
\hline Quit, but not in the next 6 months & $43(9.9 \%)$ & $31(6.7 \%)$ & $74(8.3 \%)$ \\
\hline Quit in next 6 months & $123(28 \%)$ & $153(33 \%)$ & $276(31 \%)$ \\
\hline Quit in next 30 days & $161(37 \%)$ & $216(47 \%)$ & $377(42 \%)$ \\
\hline \multicolumn{4}{|l|}{ Self-efficacy to quit } \\
\hline Impossible & $23(5.3 \%)$ & $21(4.6 \%)$ & $44(4.9 \%)$ \\
\hline Very hard & $221(51 \%)$ & $238(52 \%)$ & $459(51 \%)$ \\
\hline Hard & $148(34 \%)$ & $173(38 \%)$ & $321(36 \%)$ \\
\hline Easy & $33(7.6 \%)$ & $24(5.2 \%)$ & $57(6.4 \%)$ \\
\hline Very easy & $10(2.3 \%)$ & $5(1.1 \%)$ & $15(1.7 \%)$ \\
\hline \multicolumn{4}{|l|}{ Success to quit } \\
\hline Not at all sure & $125(29 \%)$ & $83(18 \%)$ & $208(23 \%)$ \\
\hline Slightly sure & $70(16 \%)$ & $78(17 \%)$ & $148(17 \%)$ \\
\hline Moderately sure & $137(31 \%)$ & $171(37 \%)$ & $308(34 \%)$ \\
\hline Very sure & $74(17 \%)$ & $113(25 \%)$ & $187(21 \%)$ \\
\hline Extremely sure & $29(6.7 \%)$ & $16(3.5 \%)$ & $45(5.0 \%)$ \\
\hline
\end{tabular}

$\mathrm{SD}=$ standard deviation. ${ }^{\mathrm{a}}$ Response totals may not add to group or total $N$ due to missing data. ${ }^{\mathrm{b}}$ Heaviness of Smoking Index. 
Table 4 Smoking outcomes at 8 weeks and 6.5 months.

\begin{tabular}{|c|c|c|c|c|c|c|}
\hline \multirow[b]{2}{*}{ Characteristic } & \multicolumn{2}{|c|}{$n(\%)$ or mean $(S D)$} & \multicolumn{2}{|l|}{ Crude } & \multicolumn{2}{|l|}{ Adjusted $^{\mathrm{a}}$} \\
\hline & Usual care & Intervention & Estimate $^{\mathrm{b}}(95 \% \mathrm{CI})$ & P-value & Estimate $^{b}(95 \%$ CI $)$ & P-value \\
\hline \multicolumn{7}{|l|}{8 weeks } \\
\hline Verified 7-day PPA & $8(1.8 \%)$ & $12(2.6 \%)$ & $1.49(0.3,7.2)$ & 0.622 & $1.72(0.5,5.7)$ & 0.373 \\
\hline 7-day PPA & $21(4.8 \%)$ & $39(8.5 \%)$ & $2.05(0.9,4.9)$ & 0.106 & $1.90(0.8,4.3)$ & 0.123 \\
\hline Verified continuous PPA & $5(1.1 \%)$ & $6(1.3 \%)$ & $1.20(0.3,5.0)$ & 0.803 & $1.67(0.7,4.2)$ & 0.276 \\
\hline Continuous PPA & $13(3.0 \%)$ & $16(3.5 \%)$ & $1.36(0.5,3.5)$ & 0.534 & $1.54(0.8,3.0)$ & 0.197 \\
\hline Cigarettes per day & $16(11)$ & $15(11)$ & $0.89(0.8,1.0)$ & 0.036 & $0.88(0.8,0.95)$ & 0.001 \\
\hline Number of quit attempts & $0(1)$ & $1(1)$ & $1.11(0.8,1.6)$ & 0.568 & $1.12(0.8,1.6)$ & 0.473 \\
\hline NRT used & $98(23 \%)$ & $171(37 \%)$ & $2.07(1.3,3.3)$ & 0.002 & $2.01(1.3,3.0)$ & $<0.001$ \\
\hline NRT received at the clinic & $63(14 \%)$ & $154(33 \%)$ & $3.74(1.9,7.5)$ & $<0.001$ & $3.79(2.0,7.3)$ & $<0.001$ \\
\hline \multicolumn{7}{|l|}{6.5 months } \\
\hline Verified 7-day PPA & 0 & $1(0.2 \%)$ & $1.49(0.3,7.2)$ & 0.622 & $1.72(0.5,5.7)$ & 0.373 \\
\hline 7-day PPA & $22(5.1 \%)$ & $35(7.6 \%)$ & $1.42(0.8,2.6)$ & 0.265 & $1.51(0.9,2.6)$ & 0.137 \\
\hline Verified continuous $\mathrm{PPA}^{\mathrm{c}}$ & 0 & $1(0.2 \%)$ & - & - & - & - \\
\hline Continuous PPA & $4(0.9 \%)$ & $12(2.6 \%)$ & $2.88(1.1,7.5)$ & 0.031 & $3.08(1.3,7.5)$ & 0.014 \\
\hline Cigarettes per day & $16(11)$ & $15(11)$ & $0.98(0.9,1.1)$ & 0.673 & $0.96(0.9,1.02)$ & 0.240 \\
\hline Number of quit attempts & $1(2)$ & $1(2)$ & $1.08(0.7,1.6)$ & 0.719 & $1.16(0.9,1.6)$ & 0.342 \\
\hline NRT used & $4(0.9 \%)$ & $12(2.6 \%)$ & $1.99(1.2,3.4)$ & 0.01 & $2.06(1.3,3.2)$ & 0.002 \\
\hline NRT received at the clinic & $11(2.5 \%)$ & $69(15 \%)$ & $6.78(2.7,16.9)$ & $<0.001$ & $6.48(2.6,16.2)$ & $<0.001$ \\
\hline
\end{tabular}

$\mathrm{PPA}=$ point prevalence abstinence; $\mathrm{SD}=$ standard deviation; $\mathrm{CI}=$ confidence interval; NRT = nicotine replacement therapy. ${ }^{\mathrm{a}} \mathrm{Model}$ adjusted for type of smoking policy (total versus partial/none; stratification variable), heaviness of smoking index (HSI) and type of programme (residential-rehabilitation versus other) as fixed effects; modelling for count outcomes (cigarettes per day, and number of quit attempts) also includes baseline count as a fixed effect. Crude and adjusted models include a random intercept for site to adjust for correlation of outcomes within treatment centres (clusters). ${ }^{\mathrm{b}}$ Odds ratio or incidence rate ratio. ${ }^{\mathrm{c}}$ Model fit questionable and estimates not presented.

statistically significant. This may be due to the low number of participants returning for the $\mathrm{CO}$ breath analysis, reducing power to detect differences in this outcome between groups. The self-reported 7-day abstinence rates of $8.5 \%$ for the intervention group and $4.8 \%$ for control group participants at 8 -week follow-up were also not significant. The difference in self-reported abstinence rates and verified abstinence rates is noteworthy, and highlights the importance of verifying self-report in clinical trials with this population group.

Our study showed that with support from their treatment service, smokers in the intervention group significantly reduced the number of cigarettes smoked. This may translate to cessation over a longer time-frame with longer-term NRT use. Reductions in cigarettes smoked per day are likely to have wider-reaching socio-economic impacts for the financially stressed individual. In both the addiction setting [46] and the smoke-free work-place literature [47], reduced cigarette consumption is related to the implementation of total smoking bans. On an organizational level, a smoke-free policy is recommended to support a range of smoking-related and cessation outcomes.

Significantly more participants in the intervention group used NRT, and reported that they received this cessation support from the AOD clinic they were recruited from. At the service level, if the offer and/or provision of NRT is viewed as an indicator of practice change, then we may consider the TNT intervention as successful in achieving practice change related to improving the delivery of smoking cessation care. At the client level, use of NRT is a clear indication of client interest and willingness to quit when asked and provided with appropriate cessation support in the treatment setting. Having access to and using smoking cessation medicines is associated with cessation [17]; however, on average it takes a greater number of quit attempts for vulnerable populations to quit successfully. Equipping addiction services with the training and resources to integrate smoking cessation care into their routine service provision allows for repeated opportunities for quit engagement with participants and is a clear positive outcome of the TNT intervention.

A range of types of AOD treatment programmes were included in the trial, ensuring that the results are generalizable to in- and out-patient settings and types of SUDs. However, the results are probably not generalizable to countries with health services that are different to those found in Australia.

\section{Strengths and limitations}

The trial has a number of strengths, including being, to our knowledge, the largest study of the effectiveness of 
organizational change for smoking cessation to date, the use of a pragmatic effectiveness study design, the use of an objective primary outcome measure and the first to examine the impact of the intervention on smoking cessation outcomes, rather than changes in care delivery only. The main limitation of the study is the short follow-up period. Given that one of the aims of the intervention, which was delivered over a 12-week period, was to change organizational practices and policies to deliver more smoking cessation support to clients, the 8-week and 6.5-month followup may not have been long enough to capture changes in client outcomes. Changing providers' practices is a lengthy process and the translation of that to changes in client smoking behaviours may occur in the future. The secondary outcomes captured some of the changes in practices (offering NRT). Longer-term follow-up of participants is warranted. Furthermore, we have not included in this manuscript a range of process data describing the implementation and uptake of the intervention using staff selfreport. The data describe a range of practice change outcomes (such as recording of smoking status and provision of verbal quit advice), changes in treatment service smoke-free policies and staff attitudes towards provision of smoking cessation care in SUD treatment, before and after the implementation of the intervention. The process data show significant improvements in a range of attitudes and delivery of smoking cessation practices. As this is a substantial amount of data it will be reported in an additional paper, yet to be published. Another study limitation is the high rate of attrition of participants at 8-week (53\%) and 6.5-month follow-up (48\%). However, analyses were conducted using ITT and Russell Standard principles, the attrition rate was similar to that observed in other trials with people in AOD treatment [41] and multiple types of imputation techniques were used. One of the main factors contributing to the high attrition rate was participants moving to other parts of the country following discharge from the treatment centre, making contact difficult and virtually impossible for return to the service for a breathanalysis. A number of strategies were used to boost retention, including collecting detailed contact information for the participant and a nominated family member or friend who could reach them if their details changed, maintaining regular contact between data collection time-points via reminder text messages, e-mails or letters, and offering monetary reimbursement for the completion of all assessments. Nonetheless, future research should consider additional methods for retaining participants in trials. Finally, we were unable to calculate total client eligibility, as although services were provided with recruitment logs to track number of participants approached, eligible and consenting to participate, these data have a high proportion of missing values and so were not considered suitable for inclusion in the results.

\section{CONCLUSIONS}

To our knowledge, this was the first cluster randomized controlled trial to test the impact of an organizational change intervention on improving client smoking cessation outcomes in the substance abuse setting. Although the findings in verified 7-day point prevalence abstinence were inconclusive, the organizational change intervention elicited positive behaviour change. Participants reported smoking fewer cigarettes per day and increased use of NRT provided by the AOD service to support cessation attempts. These findings indicate that smoking cessation care can be successfully integrated into routine AOD service provision through an organizational change approach. Future studies should explore impact of treatment intensity and adherence on verified smoking abstinence outcomes.

\section{Clinical trial registration details}

Trial registry: Australian New Zealand Clinical Trial Registry. Trial registration number: ACTRN12615000204549. Trial registration URL: https://www.anzctr.org.au/Trial/ Registration/TrialReview.aspx?id=367906

\section{Declaration of interests}

None.

\section{Acknowledgements}

Funding was received from the National Health and Medical Research Council (NHMRC 1045840) and the Cancer Council New South Wales (CCNSW). The NHMRC played no role in the study and the CCNSW was involved in the study advisory group. The trial was registered with the Australian and New Zealand Clinical Trials Registry (ACTRN12615000204549). Approvals were received from the Human Research Ethics Committees (HRECs) of Hunter New England Local Health District, ACT Health, SA Health and the University of Newcastle. The authors would like to thank the AOD clients who participated in this study for their time and honesty, and the CEOs, managers and staff of the Australian AOD services that participated in the research. The TNT team would like to thank the site facilitators for their part in the project.

\section{Authors' affiliations}

Faculty of Health and Medicine, School of Medicine and Public Health, University of Newcastle, Callaghan, NSW, Australia,' National Drug and Alcohol Research Centre, University of New South Wales, Randwick, NSW, Australia, ${ }^{2}$ Hunter New England Local Health District, Hunter New England Population Health, Wallsend, NSW, Australia, ${ }^{3}$ Tobacco Control Unit, Cancer Council NSW, Woolloomooloo, NSW, Australia, ${ }^{4}$ College of Health and Medicine, Australian National University, Canberra, ACT, Australia, ${ }^{5}$ Newcastle Community Health Centre, Hunter New England Local Health District, Newcastle West, NSW, Australia, ${ }^{6}$ Network of Alcohol and other Drugs Agencies, Woolloomooloo, 
NSW, Australia, ${ }^{7}$ Alcohol, Tobacco and Other Drug Association ACT, Ainslie, ACT, Australia, ${ }^{8}$ Hunter Medical Research Institute, New Lambton Heights, NSW, Australia ${ }^{9}$ and School of Psychology, University of Wollongong, Wollongong, NSW, Australia ${ }^{10}$

\section{References}

1. Guydish J., Passalacqua E., Pagano A., Martínez C., Le T., Chun J. et al. An international systematic review of smoking prevalence in addiction treatment. Addiction 2016; 111 : 220-30.

2. Australian Institute of Health and Welfare (AIHW) National Drug Strategy Household Survey. AIHW: Canberra, Australia; 2016.

3. Jamal A., Homa D. M., O'Connor E., Babb S. D., Caraballo R. S., Singh T. et al. Current cigarette smoking among adultsUnited States, 2005-2014. Morb Mortal Wkly Rep 2015; 64: 1233-40.

4. Bandiera F. C., Anteneh B., Le T., Delucchi K., Guydish J. Tobacco-related mortality among persons with mental health and substance abuse problems. PLOS ONE 2015; 10: e0120581.

5. Clarke J. G., Stein M. D., McGarry K. A., Gogineni A. Interest in smoking cessation among injection drug users. Am J Addict 2001; 10: 159-66.

6. McClure E. A., Acquavita S. P., Dunn K. E., Stoller K. B., Stitzer M. L. Characterizing smoking, cessation services, and quit interest across outpatient substance abuse treatment modalities. J Subst Abuse Treat 2014; 46: 194-201.

7. Richter K. P., Gibson C. A., Ahluwalia J. S., Schmelzle K. H. Tobacco use and quit attempts among methadone maintenance clients. Am J Public Health 2001; 91: 296.

8. Velicer W. F., Fava J. L., Prochaska J. O., Abrams D. B., Emmons K. M., Pierce J. P. Distribution of smokers by stage in three representative samples. Prev Med 1995; 24: 401-11.

9. McHugh R. K., Votaw V. R., Fulciniti F., Connery H. S., Griffin M. L., Monti P. M. et al. Perceived barriers to smoking cessation among adults with substance use disorders. J Subst Abuse Treat 2017;74:48-53.

10. Martin R. A., Cassidy R. N., Murphy C. M., Rohsenow D. J. Barriers to quitting smoking among substance dependent patients predict smoking cessation treatment outcome. J Subst Abuse Treat 2016; 64: 7-12.

11. Currie S. R., Nesbitt K., Wood C., Lawson A. Survey of smoking cessation services in Canadian addiction programs. J Subst Abuse Treat 2003; 24: 59-65.

12. Knudsen H. K., Studts C. R., Studts J. L. The implementation of smoking cessation counseling in substance abuse treatment. J Behav Health Serv Res 2012; 39: 28-41.

13. Friedmann P. D., Jiang L., Richter K. P. Cigarette smoking cessation services in outpatient substance abuse treatment programs in the United States. J Subst Abuse Treat 2008; 34: 165-72.

14. Twyman L., Bonevski B., Paul C., Bryant J. Perceived barriers to smoking cessation in selected vulnerable groups: a systematic review of the qualitative and quantitative literature. $B M J$ Open 2014; 4: e006414.

15. Prochaska J. J., Delucchi K., Hall S. M. A meta-analysis of smoking cessation interventions with individuals in substance abuse treatment or recovery. J Consult Clin Psychol 2004; 72: 1144-56.

16. Knudsen H. K. Implementation of smoking cessation treatment in substance use disorder treatment settings: a review. Am J Drug Alcohol Abuse 2017; 43: 215-25.
17. Fiore M. C., Jaén C. R., Baker T. B., Bailey W. C., Benowitz N. L., Curry S. J. et al. Treating Tobacco Use and Dependence: 2008 Update. Rockville, MD: US Department of Health and Human Services; 2008.

18. Cohn A., Elmasry H., Niaura R. Facility-level, state, and financial factors associated with changes in the provision of smoking cessation services in US substance abuse treatment facilities: results from the National Survey of substance abuse treatment services 2006 to 2012. J Subst Abuse Treat 2017; 77: 107-14.

19. Guydish J., Passalacqua E., Tajima B., Manser S. T. Staff smoking and other barriers to nicotine dependence intervention in addiction treatment settings: a review. J Psychoact Drugs 2007; 39: 423-33.

20. Knudsen H. K., Studts J. L., Boyd S., Roman P. M. Structural and cultural barriers to the adoption of smoking cessation services in addiction treatment organizations. J Addict Dis 2010; 29: 294-305.

21. Fiore M. C., Keller P. A., Curry S. J. Health system changes to facilitate the delivery of tobacco-dependence treatment. Am J Prev Med 2007; 33: S349-S356.

22. Ziedonis D. M., Zammarelli L., Seward G., Oliver K., Guydish J., Hobart M. et al. Addressing tobacco use through organizational change: a case study of an addiction treatment organization. J Psychoact Drugs 2007; 39: 451-9.

23. Guydish J., Ziedonis D., Tajima B., Seward G., Passalacqua E., Chan M. et al. Addressing tobacco through organizational change (ATTOC) in residential addiction treatment settings. Drug Alcohol Depend 2012; 121: 30-7.

24. Skelton E., Tzelepis F., Shakeshaft A., Guillaumier A., McCrabb S., Bonevski B. Integrating smoking cessation care in alcohol and other drug treatment settings using an organizational change intervention: a systematic review. Addiction 2018; 113: $2158-72$.

25. Skelton E., Tzelepis F., Shakeshaft A., Guillaumier A., Wood W., Jauncey M. et al. Integrating smoking cessation care into routine service delivery in a medically supervised injecting facility: an acceptability study. Addict Behav 2018; 84: 193-200.

26. Thomas D., Abramson M. J., Bonevski B., George J. System change interventions for smoking cessation. Cochrane Database Syst Rev 2017; 10: CD010742.

27. Skelton E., Tzelepis F., Shakeshaft A., Guillaumier A., Wood W., Jauncey M. et al. Integrating smoking cessation care into a medically supervised injecting facility using an organizational change intervention: a qualitative study of staff and client views. Int J Environ Res Public Health 2019; 16: 2050 .

28. Bonevski B., Guillaumier A., Shakeshaft A., Farrell M., Tzelepis F., Walsberger S. et al. An organizational change intervention for increasing the delivery of smoking cessation support in addiction treatment centres: study protocol for a randomized controlled trial. Trials 2016; 17: 290.

29. Freund M., Campbell E., Paul C., Sakrouge R., Lecathelinais C., Knight J. et al. Increasing hospital-wide delivery of smoking cessation care for nicotine dependent in patients: a multi-strategic intervention trial. Addiction 2009; 104: 839-49.

30. Skelton E., Bonevski B., Tzelepis F., Shakeshaft A., Guillaumier A., Dunlop A. et al. Tobacco smoking policies in Australian alcohol and other drug treatment services, agreement between staff awareness and the written policy document. BMC Public Health 2017; 17: 87. 
31. Ahluwalia J. S., Gibson C. A., Kenney R. E., Wallace D. D., Resnicow K. Smoking status as a vital sign. J Gen Intern Med 1999; 14: 402-8.

32. Carson K. V., Verbiest M. E., Crone M. R., Brinn M. P., Esterman A. J., Assendelft W. J. et al. Training health professionals in smoking cessation. Cochrane Database Syst Rev 2012; 5: CD000214.

33. Bonevski B., Sanson-Fisher R., Campbell E., Carruthers A., Reid A., Ireland M. Randomized controlled trial of a computer strategy to increase general practitioner preventive care. Prev Med 1999; 29: 478-86.

34. Hatsukami D. K., Stead L. F., Gupta P. C. Tobacco addiction. Lancet 2008; 371: 2027-38

35. Hughes J. R., Keely J. P., Niaura R. S., Ossip-Klein D. J., Richmond R. L., Swan G. E. Measures of abstinence in clinical trials: issues and recommendations. Nicotine Tob Res 2003; 5: $13-25$.

36. Society for Research on Nicotine and Tobacco (SRNT) Subcommittee on Biochemical Verification Biochemical verification of tobacco use and cessation. Nicotine Tob Res 2002; 4: 149-59.

37. West R., Hajek P., Stead L., Stapleton J. Outcome criteria in smoking cessation trials: proposal for a common standard. Addiction 2005; 100: 299-303.

38. Heatherton T. F., Kozlowski L. T., Frecker R. C., Fagerstrom K. $\mathrm{O}$. The Fagerström test for nicotine dependence: a revision of the Fagerstrom tolerance questionnaire. Br J Addict 1991; 86: 1119-27.

39. Bryant J., Bonevski B., Paul C. A survey of smoking prevalence and interest in quitting among social and community service organization clients in Australia: a unique opportunity for reaching the disadvantaged. BMC Public Health 2011; 11: 827.

40. Bryant J., Bonevski B., Paul C., Lecathelinais C. Assessing smoking status in disadvantaged populations: is computer administered self report an accurate and acceptable measure? BMC Med Res Methodol 2011; 11: 153 .
41. Teesson M., Mills K., Ross J., Darke S., Williamson A., Havard A. The impact of treatment on 3 years' outcome for heroin dependence: findings from the Australian treatment outcome study (ATOS). Addiction 2008; 103: 80-8.

42. Teesson M., Ross J., Darke S., Lynskey M., Ali R., Ritter A. et al. One year outcomes for heroin dependence: findings from the Australian treatment outcome study (ATOS). Drug Alcohol Depend 2006; 83: 174-80.

43. Little R. J. A. Pattern-mixture models for multivariate incomplete data. J Am Stat Assoc 1993; 88: 125-34.

44. Rubin D. B. Multiple Imputation for Non-response in Surveys. New York, USA: John Wiley \& Sons; 2004.

45. Thurgood S. L., McNeill A., Clark-Carter D., Brose L. S. A systematic review of smoking cessation interventions for adults in substance abuse treatment or recovery. Nicotine Tob Res 2016; 18: 993-1001.

46. Guydish J., Yip D., Le T., Gubner N. R., Delucchi K., Roman P. Smoking-related outcomes and associations with tobaccofree policy in addiction treatment, 2015-2016. Drug Alcohol Depend 2017; 179: 355-61.

47. Fichtenberg C. M., Glantz S. A. Effect of smoke-free workplaces on smoking behaviour: systematic review. BMJ 2002; 325: 188.

\section{Supporting Information}

Additional supporting information may be found online in the Supporting Information section at the end of the article.

Data S1 Statistical Analysis Plan.

Table S1 Tackling Nicotine Together intervention core components.

Table S2 Sensitivity analyses: Complete case analysis, and MI-MNAR sensitivity analyses (primary outcome only). 